\title{
Oridonin inhibition and miR-200b-3p/ZEB1 axis in human pancreatic cancer
}

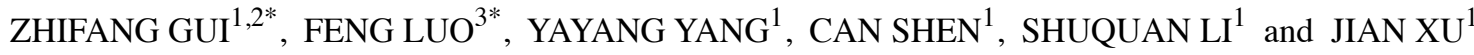 \\ ${ }^{1}$ Medical Technology College, Zhejiang Chinese Medical University, Hangzhou, Zhejiang 310053; \\ ${ }^{2}$ Department of Clinical Laboratory, The Affiliated Hospital of Hangzhou Normal University, \\ Hangzhou, Zhejiang 310015; ${ }^{3}$ Department of Clinical Laboratory, The First Affiliated Hospital \\ of Zhejiang Chinese Medical University, Hangzhou, Zhejiang 310006, P.R. China
}

Received August 8, 2016; Accepted November 10, 2016

DOI: $10.3892 /$ ijo.2016.3772

\begin{abstract}
The relationship among oridonin, miR-200b-3p and pancreatic cancer on epithelial-to-mesenchymal transition (EMT) was investigated for the molecular mechanism or signaling pathways on the migration in pancreatic cancer. BxPC-3 and PANC-1 cells were cultivated and the $\mathrm{IC}_{50}$ of oridonin in BxPC-3 and PANC-1 cells were obtained by the CCK-8 array. The expression of miR-200b-3p was verified by using real-time PCR and its target gene was predicted. BxPC-3 and PANC-1 cells were treated with oridonin or transfected by miR-200b-3p, those cells were used for western blot assay, Transwell assay, ELISA, immunofluorescence staining, tumorigenesis assay in nude mice and immunohistochemical assay to verify the effects of oridonin or miR-200b-3p on pancreatic cancer. We found that oridonin inhibited the proliferation of BxPC-3 and PANC-1 cells in a dose-dependent manner. miR-200b-3p was downregulated by oridonin in BxPC-3 and PANC-1 cells. ZEB1 was a target gene for miR-200b-3p. Oridonin or overexpression of miR-200b-3p can inhibit the cell migration in BxPC-3 and PANC-1 cells. miR-200b-3p can inhibit the EMT and oridonin can inhibit the expression of ZEB1, N-cadherin and fibronectin but not increase the expression of E-cadherin, while the cell adhesion molecules ICAM-1 and VCAM-1 were decreased by oridonin in BxPC-3 and PANC-1 cells and the cytoskeleton was altered by oridonin in PANC-1 cells compared with the control. In summary, the results demonstrate that miR-200b-3p was able to inhibit the EMT of human pancreatic cancer in vivo and in vitro by targeted ZEB1. In vitro, oridonin had a certain
\end{abstract}

Correspondence to: Professor Jian Xu, Medical Technology College, Zhejiang Chinese Medical University, Hangzhou, Zhejiang 310053, P.R. China

E-mail: xujian832002@163.com

${ }^{*}$ Contributed equally

Key words: oridonin, miR-200b-3p, pancreatic cancer, epithelialto-mesenchymal transition, migration effect on the migration in BxPC-3 and PANC-1 cells, but not though type III EMT by miR-200-3p/ZEB1 axis, and may be related to type II EMT, tumor microenvironment or altering the cytoskeleton. In vivo, oridonin inhibited the cancer migration in the nude mouse model though inhibiting EMT.

\section{Introduction}

Pancreatic cancer is a one of the most malignant digestive system cancers with a high morbidity and mortality in both developed and developing countries. Pancreatic cancer is characterized by no early detectable biomarkers, strong resistance to chemotherapy and radiation therapy, highly metastatic potential, worst prognosis and only $5 \%$ of 5 -year survival rate $(1,2)$. Therefore, searching for new safer treatments for pancreatic cancer is essential.

Recently, traditional Chinese medicines have become a 'hot spot' for its ability to simultaneously address multiple targets, no side-effects to patients, improving the sensitivity of chemotherapy and radiation therapy and enhancing immunity. Oridonin, extracted from Rabdosia rubescens, is a natural ent-kaurane diterpenoid compound, which has many physiological and pharmacological effects, including heat-clearing, detoxifying, anti-inflammatory and anti-tumorigenicity (3). Previous studies have reported that oridonin can inhibit proliferation, induce cell cycle arrest and apoptosis and metastasis in many cancers (4-7), but the definite molecular action mechanisms of inhibition of pancreatic cancer have not yet been clarified.

MicroRNAs (miRNAs) are highly conserved, small noncoding RNAs with lengths of 17-25 nucleotides (nt), which play important gene-regulatory roles in animals and plants by pairing with target protein coding genes at the post-transcriptional level (8). An increasing number of studies indicate that miRNAs play crucial roles in many important biological processes, including development, proliferation, apoptosis, immune response and even tumorigenesis (9). There are more than 15,000 miRNAs found in over 140 species (10), while most of their functions have not yet been defined and validated. The miR-200 family is known to be involved in the epithelial-tomesenchymal transition (EMT), a complex biological process, in which polarized epithelial cells convert into a mesenchymal 
phenotype, epithelial cells lose their epithelial characteristics and acquire motile mesenchymal properties: loss of cellcell adhesion; increased motility and invasiveness $(11,12)$. During the acquisition of EMT characteristics, cancer cells lose the epithelial markers, such as E-cadherin, and gain the mesenchymal markers, such as vimentin, fibronectin and $\mathrm{N}$-cadherin (13). EMT plays key roles in many physiological and pathological process, such as embryonic development, wound healing and carcinogenesis (14). EMT was recently classified as type III EMT: type I EMT is during implantation, embryogenesis and organ development; type II EMT is associated with tissue regeneration and organ fibrosis; type III EMT is associated with cancer progression and metastasis (15). The miR-200b-3p is a member of the miR-200 family, which has been demonstrated to play an important role in migration and invasion in many types of cancer, such as cervical (16), breast cancer (17) and esophageal squamous cell carcinoma (18). However, the specific role and molecular mechanism of miR-200b-3p in human pancreatic cancer remain poorly understood.

In the present study, the relationship among oridonin, miR-200b-3p and pancreatic cancer on EMT were investigated to look for the molecular mechanism or signaling pathways on the migration in pancreatic cancer in order to find new safer treatments for the pancreatic cancer.

\section{Materials and methods}

Cell culture. The BxPC-3 and PANC-1 human pancreatic cancer cell lines were provided by the Institute of Biochemistry and Cell Biology, Shanghai Institute of Biological Sciences, Chinese Academy of Sciences. The cells were respectively cultured in RPMI-1640 (BxPC-3) (Gibco, Grand Island, NY, USA) or Dulbecco's modified Eagle's medium (DMEM; Gibco) culture medium containing $10 \%$ fetal bovine serum (FBS; Gibco) in a humidified incubator with $5 \% \mathrm{CO}_{2}$ at $37^{\circ} \mathrm{C}$. Exponentially growing cells were used for the experiments.

Cell viability assay. Cell viability was quantified using Cell Counting kit-8 (CCK-8; Beyotime Institute of Biotechnology, Shanghai, China) according to the manufacturer's instructions. BxPC- 3 cells $\left(1 \times 10^{4}\right.$ cells/well) and PANC- 1 cells $\left(5 \times 10^{3}\right.$ cells/ well) were respectively seeded into 96 -well plates in a final volume of $100 \mu \mathrm{l}$ and cultured for $24 \mathrm{~h}$. Then, the medium was replaced by $100 \mu \mathrm{l}$ culture medium containing different concentrations of oridonin $(0,20,40,60,80,100,120,140$ or $160 \mu \mathrm{M})$ (Gracia Chemical Technology Company, Ltd., Chengdu, China; $98 \%$ purity, HPLC), and the cells were incubated for $24 \mathrm{~h}$. The negative control cells were treated with medium containing $0.1 \%$ dimethyl sulfoxide (DMSO) only. The same DMSO concentration was used for drug delivery. After oridonin treatment, $10 \mu \mathrm{l}$ of CCK- 8 solution was added into each well and the cells were incubated for $4 \mathrm{~h}$ at $37^{\circ} \mathrm{C}$. The absorbance was measured at $450 \mathrm{~nm}$ using a microplate reader (Model 680; Bio-Rad Laboratories, Hercules, CA, USA) and the percentage of cell viability was calculated as follows: Viability ratio $\%=\mathrm{A} 450$ (oridonin)/A450(control) $\times 100 \%$. The $\mathrm{IC}_{50}$ value was calculated as the concentration of oridonin that inhibited cell growth by $50 \%$. At least 3 independent experiments were performed.
RNA isolation, quantitative real-time PCR and target prediction. BxPC-3/ PANC-1 cells in logarithmic growth phase were seeded in $60-\mathrm{mm}$ dishes at a density of $2 \times 10^{5}$ cell/well and incubated overnight. One group of these cells was subsequently treated with $87.8 \mu \mathrm{M}$ (BxPC-3)/55.8 $\mu \mathrm{M}$ (PANC-1) oridonin and another was used as a blank control group cultured in medium containing $0.1 \%$ DMSO for $24 \mathrm{~h}$. Total RNA (containing small RNAs) was extracted using the TRIzol LS reagent (Invitrogen/Life Technologies) following the manufacturer's protocol. cDNA was synthesized in a MyCycler ${ }^{\mathrm{TM}}$ Thermal Cycler (Bio-Rad Laboratories), and miR-200b-3p levels were quantified by quantitative real-time polymerase chain reaction (qPCR) in a real-time PCR detector (Bio-Rad Laboratories) using the PrimeScript ${ }^{\mathrm{TM}}$ miRNA qPCR Starter kit ver.2.0 (Takara, Dalian, China) following the manufacturer's protocol, and calculated using RNU6B as the internal control by the $2^{-\Delta \Delta C T}$ method. The $5^{\prime}-3^{\prime}$ sequence of the primer miR-200b-3p is TAATACTGCCTGGTAATGATGA. All of the reactions were run in triplicate. In order to find the functional explanation of miR-200b-3p, we explored the most potential targets of miR-200b-3p. The prediction of miR-200b-3p targets was performed using online software TargetScan (http://www.targetscan.org/), PicTar (http://pictar. mdc-berlin.de/cgi-bin/new_PicTar_vertebrate.cgi) and miRanda (http://www.microrna.org/microrna/home.do). The intersection of the results from these three types of software was taken as the final target genes.

Oligonucleotide transfection. The miR-200b-3p mimic, the mimics negative control precursor (NC), inhibitor and an inhibitor negative control precursor (NC) were obtained from Shanghai GenePharma, Co., Ltd. (Shanghai, China). These transfections were performed using Lipofectamine ${ }^{\mathrm{TM}} 2000$ (Invitrogen, Carlsbad, CA, USA) following the manufacturer's protocol. Cells were grown in 6-well culture plates at a density of $2 \times 10^{5}$ (BxPC-3) $/ 3 \times 10^{5}$ (PANC-1) cells/well for $24 \mathrm{~h}$. For each well, $5 \mu$ l human miR-200b-3p mimics/inhibitor or mimics/ inhibitor inhibitor $\mathrm{NC}$ was added to $250 \mu \mathrm{l}$ culture medium. Next, $5 \mu \mathrm{l}$ of Lipofectamine 2000 was added to $250 \mu \mathrm{l}$ culture medium and then mixed. The mixture was added to the cells, incubated for $6 \mathrm{~h}$ and then was replaced by $2000 \mu \mathrm{l}$ of new culture medium. For western blot assays, cells were collected $48 \mathrm{~h}$ after the transfection.

Western blot analysis. Total protein was extracted from cells or tissues. BxPC-3 or PANC-1 cells treated with oridonin or transfected by miR-200b-3p were rinsed twice with phosphate-buffered saline (PBS) and lysed in RIPA lysis buffer (Beyotime Institute of Biotechnology) along with PMSF. The lysates were centrifuged, and the supernatants were collected to be quantitated using a bicinchoninic acid assay kit (PQ0011; Multi Sciences Biotech, Co., Ltd., Hangzhou, China). A total of $40 \mu \mathrm{g}$ of proteins was loaded onto an $8 \%$ SDS-polyacrylamide gel for electrophoresis followed by transfer to polyvinylidene difluoride (PVDF) membranes. Skim milk (5\%) in Tris-buffered saline and Tween-20 was used to block the membrane for $2 \mathrm{~h}$ at room temperature. Then, the membrane was incubated overnight at $4^{\circ} \mathrm{C}$ with the following primary antibody: TCF8/ZEB1 (D80D3) rabbit mAb (1:1,000, \#3396; Cell SignalingTechnology, Danvers, 
MA, USA), E-cadherin (24E10) rabbit mAb (1:1,000, \#3195; Cell Signaling Technology), anti-fibronectin antibody (1:1,000, ab2413; Abcam), anti-N cadherin antibody [5D5] (1:1,000, ab98952; Abcam), anti- $\beta$-actin monoclonal antibody (1:1,000, Mab1445; Multi Sciences Biotech). The membranes were then incubated with secondary antibodies conjugated with horseradish peroxidase (HRP): goat anti-mouse IgG, (GAM0072; Multi Sciences Biotech) or goat anti-rabbit IgG (GAR0072 Multi Sciences Biotech) for $2 \mathrm{~h}$ at room temperature. The membranes were then visualized using an ECL substrate kit (P1425; Multi Sciences Biotech) on the Omega Lum G Imaging System. $\beta$-actin levels were used to standardize protein loading. ImageJ software was used to quantify band intensities. All analyses were conducted in triplicate.

Lentivirus transduction. The lentiviral LV-miR-200b-3p and LV-NC (miR-200b-3p-expressing or vector-control lentivirus) was purchased from Shanghai GenePharma. Lentivirus transfections were performed according to the manufacturer's instructions to establish anti-miR-200b-3p-expressing stable clones in BxPC-3 and PANC-1 cells (LV-miR-200b-3p). The control clones (LV-NC) were produced with a similar method. Briefly, the BxPC- 3 cells $\left(2 \times 10^{5}\right)$ or PANC- 1 cells $\left(1 \times 10^{5}\right)$ were seeded in each 24 -well culture plate for $24 \mathrm{~h}$ and then were infected with LV-miR-200b-3p or LV-NC in the presence of $5 \mu \mathrm{g} / \mathrm{ml}$ polybrene and incubated for $24 \mathrm{~h}$. After replaced by $2000 \mu \mathrm{l}$ of new culture medium, the cells were cultured for another $48 \mathrm{~h}$. The cells were selected using $2 \mu \mathrm{g} / \mathrm{ml}($ BxPC-3) and $1 \mu \mathrm{g} / \mathrm{ml}$ (PANC-1) puromycin to establish stable cell lines.

Migration assays. The migration assays was performed using 24-well Transwell chambers ( $8 \mu \mathrm{m}$; Millpore Corp., Billerica, MA, USA). (BxPC-3) or $3 \times 10^{4}$ (PANC-1) cells $\left(4 \times 10^{4}\right)$ or treated with oridonin, miR-200b-3p or NC in $200 \mu \mathrm{l}$ medium without FBS were seeded in the upper chamber of Transwell migration chambers without Matrigel ( $8 \mu \mathrm{m}$; Millpore Corp.). The lower chamber was filled with $500 \mu$ l culture medium containing 10\% FBS. After $24 \mathrm{~h}$, the upper chamber was washed twice with 1X PBS and fixed by $4 \%$ polyformaldehyde for $30 \mathrm{~min}$. The cells remaining on the upper membrane were removed with cotton wool, whereas the cells that had migrated through the membrane were stained with $0.1 \%$ crystal violet for $30 \mathrm{~min}$, or DAPI $(1 \mu \mathrm{g} / \mathrm{ml})$ for $15 \mathrm{~min}$. After three washes with PBS, the samples were photographed and counted in five independent $\mathrm{x} 400$ fields for each well using an microscope (Olympus, Tokyo, Japan). The mean values of the data were obtained from three separate chambers.

Detection of ICAM-1 and VCAM-1 production. The levels of ICAM-1 and VCAM-1 in response to treatment of cells with oridonin $(0$, or $87.8 \mu \mathrm{M} / 55.8 \mu \mathrm{M})$ was determined using a human enzyme linked immunosorbent assay (ELISA) kits. Briefly, culture supernatants of cells were collected at $24 \mathrm{~h}$ post-treatment and cleared of cell debris by brief centrifugation. ICAM-1 and VCAM-1 levels were measured using human intercellular adhesion molecule 1, ICAM-1 ELISA kit (CSB-E04574h; Cusabio Biotech, Co., Ltd., Wuhan, China) and human vascular cell adhesion molecule 1, VCAM-1 ELISA kit (CSB-E04753h; Cusabio Biotech) according to the manufacturer's instructions. The absorbance was measured at $490 \mathrm{~nm}$ using a microplate reader.

Immunofluorescence and confocal microscopy. For immunofluorescence experiments, PANC-1 cells were plated onto 35-mm glass-based dishes (801002; NEST Biotechnology, New Orleans, LA, USA) glass one day before the treatment with oridonin ( 0 or $55.8 \mu \mathrm{M}$ ) for $24 \mathrm{~h}$. Cells were fixed in $4 \%$ polyformaldehyde for $30 \mathrm{~min}$ and permeabilized with $0.2 \%$ Triton X-100 for 15 min. Then, they were blocked with 5\% BSA for $1 \mathrm{~h}$. Primary antibody incubations with the antibody $\beta$-tubulin (1:200, ab009; Multi Sciences Biotech) in PBS containing $2 \% \mathrm{FBS}$ were performed overnight at $4^{\circ} \mathrm{C}$ followed by incubation with the goat anti-mouse IgG H\&L (DyLight 488) (1:400, ab96871; Abcam) in PBS containing 2\% FBS as a secondary antibody for $2 \mathrm{~h}$ at room temperature. Finally, cell nuclei were stained with DAPI $(0.5 \mu \mathrm{g} / \mathrm{ml})$ for $15 \mathrm{~min}$, and images were obtained using a confocal fluorescence microscope. PBS was used for all washing steps.

Tumor formation assay in nude mice. The $16 \mathrm{BALB} / \mathrm{C}$ nude mice (5-week-old, 20-25 g body weight) were provided from the Animal Experimental Center of the Zhejiang Chinese Medical University in accordance with the Institutional policies. The mice were randomly divided into four groups ( $n=4 /$ group): the control group, the oridonin group, the miR-200b-3p group and the NC group. The control group and the oridonin group were injected with $5 \times 10^{6}$ BxPC-3 cells; the miR-200b-3p group and the NC group were injected with $5 \times 10^{6}$ BxPC-3 cells infected with miR-200b-3p or mock vector, respectively. These cells were all suspended in $200 \mu 1$ RPMI-1640 medium and injected subcutaneously into the flank of each nude mice. When the volume of tumor reached $150 \mathrm{~mm}^{3}$, oridonin was injected into the mice of the oridonin group by intraperitoneal injection at a concentration of $10 \mathrm{mg} / \mathrm{kg}$ and the other groups were injected with the same amount of saline every day. Tumor sizes and body weights were measured two or three times per week. The tumor volume was calculated as follows: Tumor volume $\left(\mathrm{mm}^{3}\right)=1 / 2 \mathrm{x}$ length $\mathrm{x}$ width ${ }^{2}$. After 7 weeks, the mice were sacrificed and the xenograft tumors were excised, weighed, harvested and fixed for the next experiment.

$H \& E$ staining and immunohistochemistry. For histopathologic analysis, tumor sections from the control group and the normal pancreas from the nude mice were fixed in $10 \%$ paraformaldehyde and embedded in paraffin for hematoxylin and eosin staining. Immunohistochemistry analysis (IHC) was performed as per the manufacturer's protocol of Histostain ${ }^{\mathrm{TM}}$ -Plus kits (Bioss, Beijing, China). In brief, mouse tumor tissues were fixed in $10 \%$ paraformaldehyde and embedded in paraffin, and antigen retrieval was carried out using a target retrieval solution. The sections were incubated with a rabbit antibody to E-cadherin (\#3195; 1:100; Cell Signaling Technology) or vimentin (\#5741; 1:100; Cell Signaling Technology) overnight at $4^{\circ} \mathrm{C}$, followed by another incubation with a horseradish peroxidase-conjugated goat-anti-rabbit secondary antibody for $2 \mathrm{~h}$ at $37^{\circ} \mathrm{C}$. Finally, the sections were counterstained with hematoxylin and images (x20) were acquired with a microscope. 

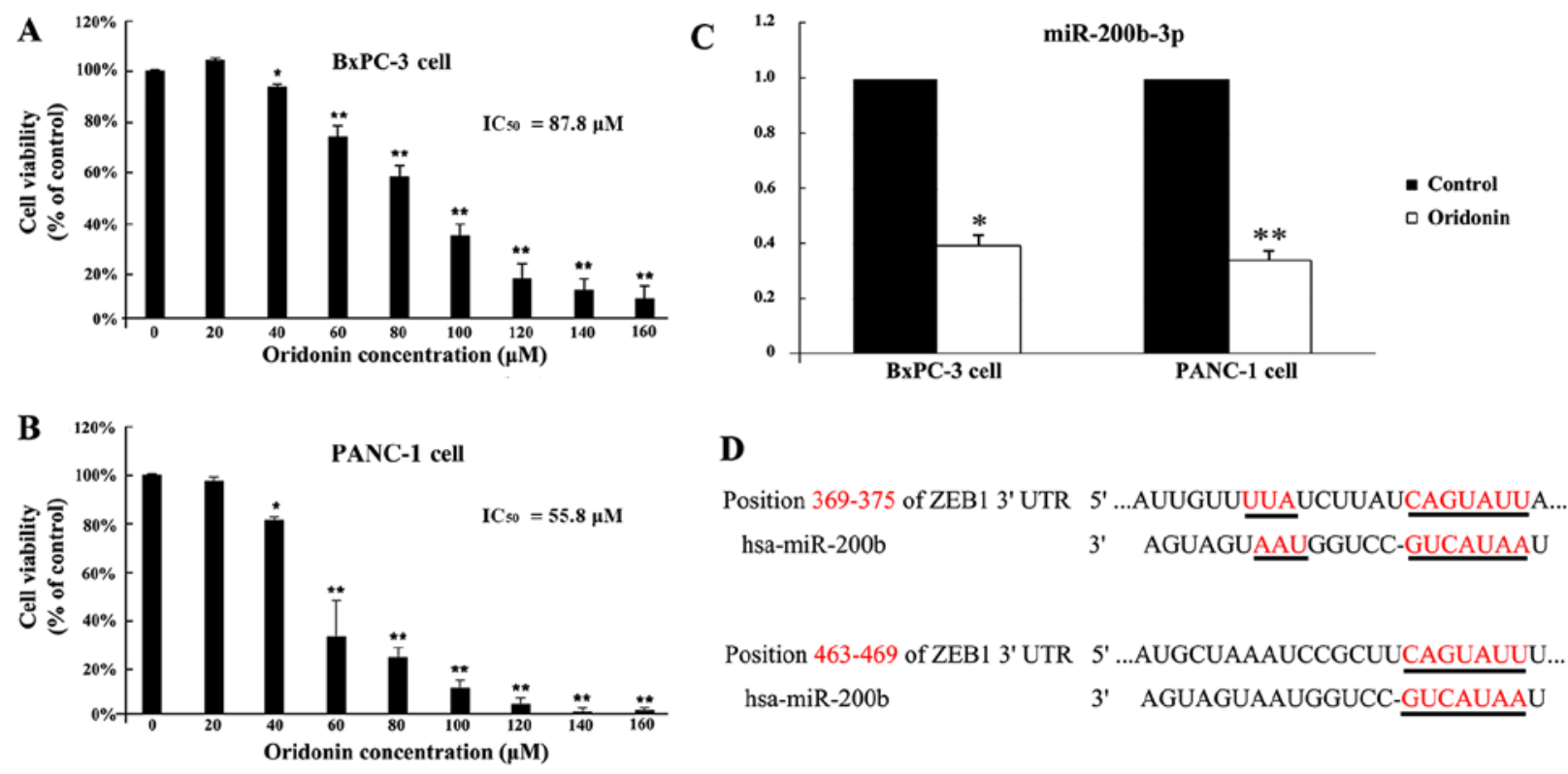

Figure 1. Oridonin inhibits cell proliferation and alters miR-200b-3p expression in BxPC-3 and PANC-1 cells. (A and B) BxPC-3 and PANC-1 cells were treated with $0.1 \%$ DMSO or $0.1 \%$ DMSO containing various concentrations of oridonin for $24 \mathrm{~h}$. The percentage of cell viability in each sample was determined using an CCK-8 assay. Cytotoxicity was expressed as the percentage of cell inhibition compared with the control (DMSO only). (C) Relative miR-200b-3p expression levels in BxPC-3 and PANC-1 cells treated with oridonin vs. the control. The expression levels were measured by qRT-PCR. (D) Putative miR-200b-3p binding sequences in the 3'-UTR of ZEB1 mRNA. Data are expressed as mean $\pm \mathrm{SD},{ }^{*} \mathrm{P}<0.05$ and ${ }^{* * *} \mathrm{P}<0.01$.

Statistical analysis. Statistical analysis was performed using Statistical Program for Social Sciences (SPSS) software 19.0. The results from at least three independent experiments are presented as means \pm SD. Two different groups were analyzed using the two-tailed Student's t-test. All tests performed were two-sided. $\mathrm{P}<0.05$ and $\mathrm{P}<0.001$ were considered statistically significant.

\section{Results}

Oridonin affects cell viability and inhibits cell proliferation. To investigate the effect of oridonin on viability in BxPC-3 and PANC-1 cells, cells were treated with various concentrations of oridonin for $24 \mathrm{~h}$. As shown in Fig. 1A and B, oridonin inhibited BxPC-3 and PANC-1 cell proliferation in a dose-dependent manner. At very low doses $(20 \mu \mathrm{M})$, oridonin showed no significant difference with the control cells in BxPC-3 and PANC-1 cells. The cell proliferation was inhibited in BxPC-3 and PANC-1 cells following treatment with $40 \mu \mathrm{M}$ oridonin compared to the control $(\mathrm{P}<0.05)$. The $50 \%$ inhibitory concentration of oridonin $\left(\mathrm{IC}_{50}\right)$ was $87.8 \mu \mathrm{M}$ in BxPC-3 cells and 55.8 $\mu \mathrm{M}$ in PANC-1 cells by using SPSS 19.0 software. Then, BxPC-3 and PANC-1 cells were treated with oridonin at its $\mathrm{IC}_{50}$ value.

Oridonin treatment alters miR-200b-3p expression in $B x P C-3$ and PANC-1 cells. A previous microarray study has shown that 105 miRNAs are differentially expressed in BxPC-3 cells treated with oridonin at concentrations of 0 and $87.8 \mu \mathrm{M}$ (19). As shown in Fig. 1C, the qPCR verified results showed that miR-200b-3p was downregulated 2.22-fold $(\mathrm{P}<0.05)$. Furthermore, we have validated the results in another human pancreatic cancer cell line PANC-1. In PANC-1 cells, oridonin treatment also downregulated the expression levels of miR-200b-3p $(\mathrm{P}<0.01)$. A miRNA usually exerts its function by suppressing the expression of target genes. Therefore, our next aim was to investigate the targets of miR-200b-3p using three different types of online software: TargetScan, PicTar and miRanda. The intersection of the three software predictions was taken as the final potential target genes. Based on the bioinformatics prediction that there are two potential positions, 369-375 and 463-469, in the 3'UTR of ZEB1 mRNA targeted by miR-200b-3p (Fig. 1D). These results suggested that miR-200b-3p is downregulated in human pancreatic cancer BxPC-3 and PANC-1 cells with oridonin treatment, and may be a potential therapeutic target of oridonin for pancreatic cancer, although further studies are required to explore this possibility.

Oridonin/miR-200b-3p regulate expression of proteins on EMT in BXPC-3 and PANC-1 cells. To verify the candidate genes regulated by miR-200b-3p, we subsequently compared ZEB1 protein levels in cells transfected with miR-200b-3p and the control by western blot analysis. As shown in Fig. 2, ZEB1 protein levels were significantly downregulated in BxPC-3 and PANC-1 cells transfected with miR-200b-3p mimics, but not in those transfected with mimics NC. These results demonstrated that ZEB1 is a target of miR-200b-3p. We next examined the effect of oridonin/miR-200b-3p on EMT in BxPC-3 and PANC-1 cells. BxPC-3 and PANC-1 cells were treated with oridonin at its $\mathrm{IC}_{50}$ or transfected with miR-200b-3p for western blot analysis. The results showed that the protein expression levels of ZEB1, E-cadherin, N-cadherin and fibronectin decreased in the BxPC-3 and PANC-1 cells treated with oridonin, comparing to the control. miR-200b-3p overexpression significantly suppressed ZEB1, N-cadherin and fibronectin expression and increased the expression of E-cadherin (Fig. 2). 


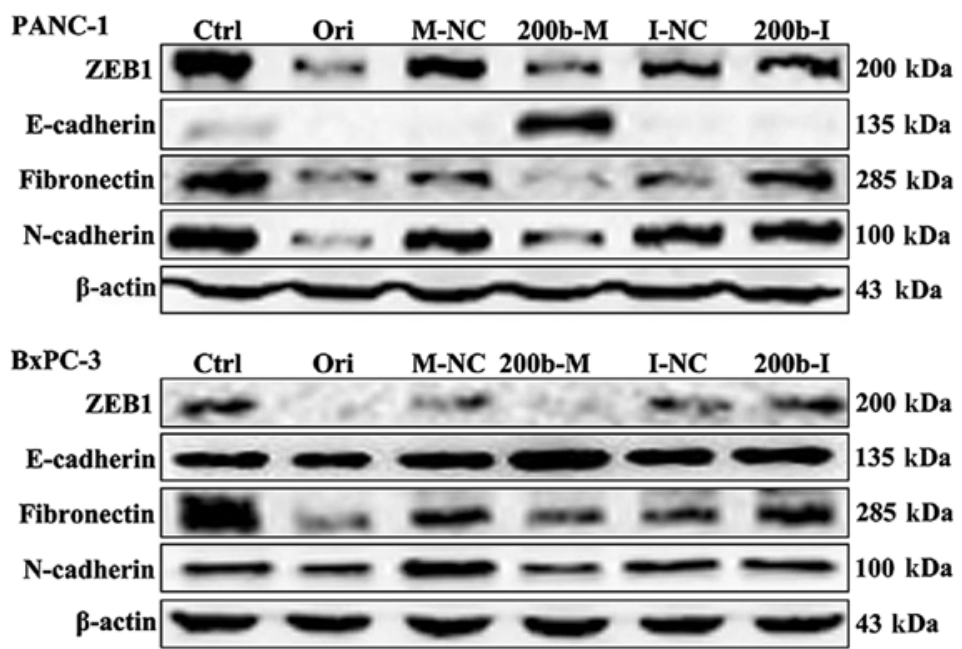

Figure 2. Oridonin/miR-200b-3p regulates the expression of proteins on EMT. Western blot analysis of the effect of oridonin/miR-200b-3p on protein levels of ZEB1, E-cadherin, N-cadherin and fibronectin in BxPC-3 and PANC-1 cells. Ctrl, control; Ori, oridonin; M-NC, mimics NC; 200b-M, miR-200b-3p mimics; I-NC, inhibitor NC; 200b-I, miR-200b-3p inhibitor.

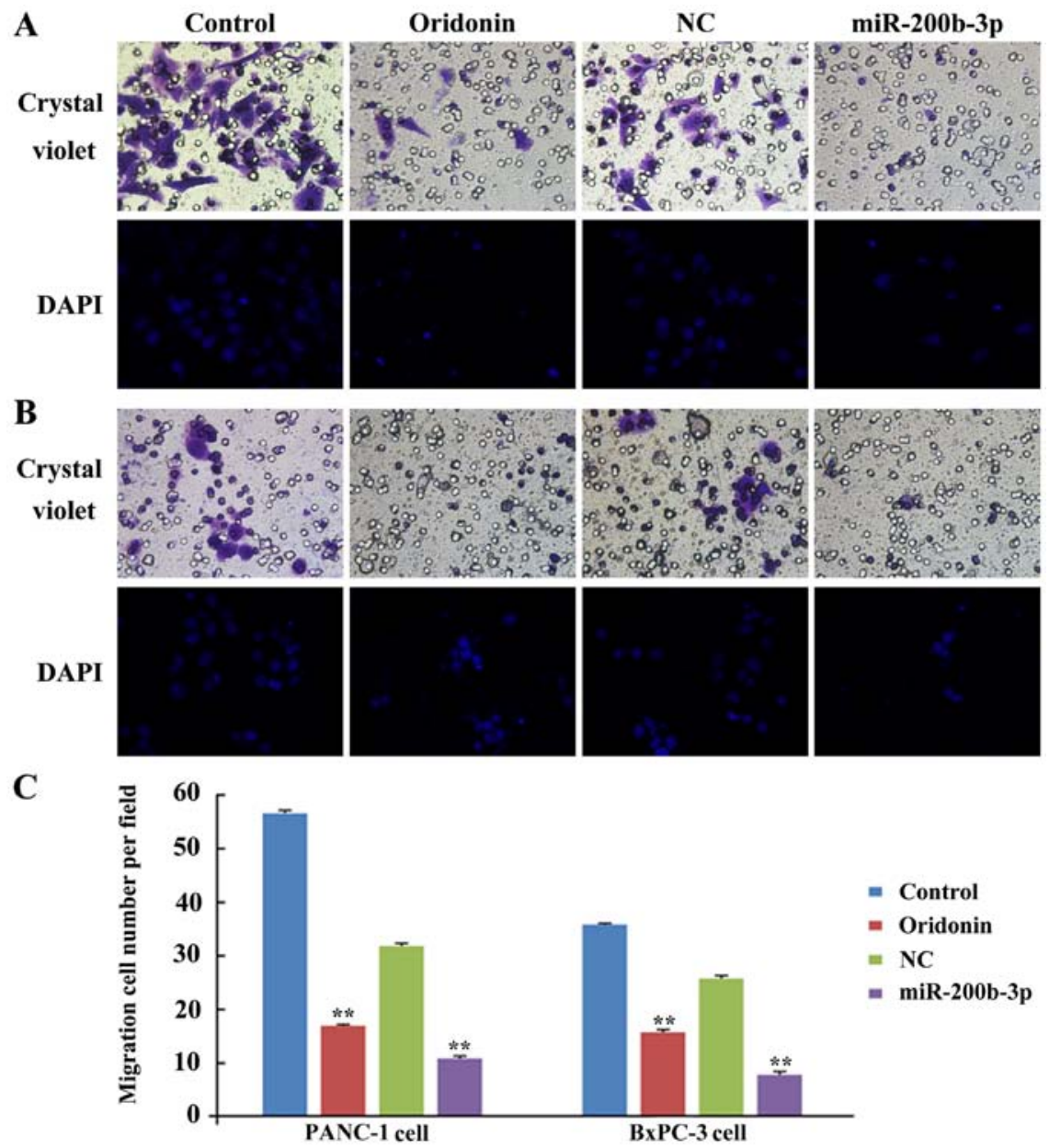

Figure 3. Inhibitory effects of oridonin/miR-200b-3p on cell migration. (A) The effect of oridonin/miR-200b-3p on migration in BxPC-3 cells; (B) the effect of oridonin/miR-200b-3p on migration in PANC-1 cells; (C) the number of migration cells; Control, the control group; oridonin, the cells treated with oridonin; $\mathrm{NC}$, cells transfected with the vector-control lentivirus; miR-200b-3p, cells transfected with the miR-200b-3p-expressing lentivirus; ${ }^{* *} \mathrm{P}<0.01$ vs. control.

Oridonin/miR-200b-3p inhibit the migration of BxPC-3 and PANC-1 cells. To study the role of oridonin/miR-200b-3p in cell migration, we assessed the capacity of migration in
BxPC-3 and PANC-1 cells after treatment with oridonin or transfection with a miR-200b-3p lentivirus by using crystal violet stain and DAPI. As shown in Fig. 3, much fewer cells 

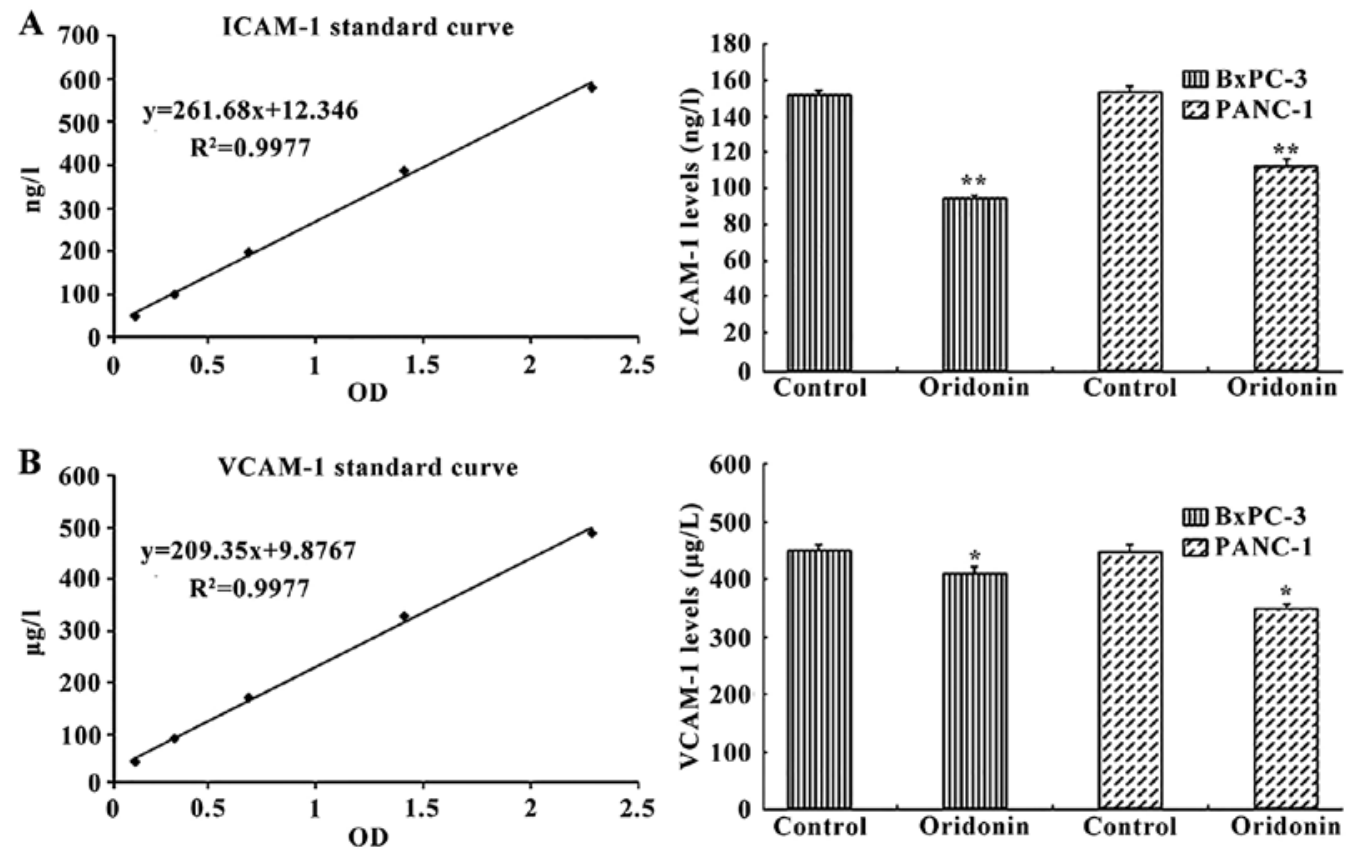

Figure 4. Oridonin decreases both the expression of ICAM-1 and VCAM-1 in BxPC-3 and PANC-1 cells. (A) Enzyme-linked immunosorbent assay analysis of the effect of oridonin on ICAM-1 in BxPC-3 and PANC-1 cells. (B) Enzyme-linked immunosorbent assay analysis of the effect of oridonin on VCAM-1 in BxPC-3 and PANC-1 cells. Data are expressed as mean \pm SD, ${ }^{*} \mathrm{P}<0.05$ vs. control, ${ }^{* *} \mathrm{P}<0.01$ vs. control.
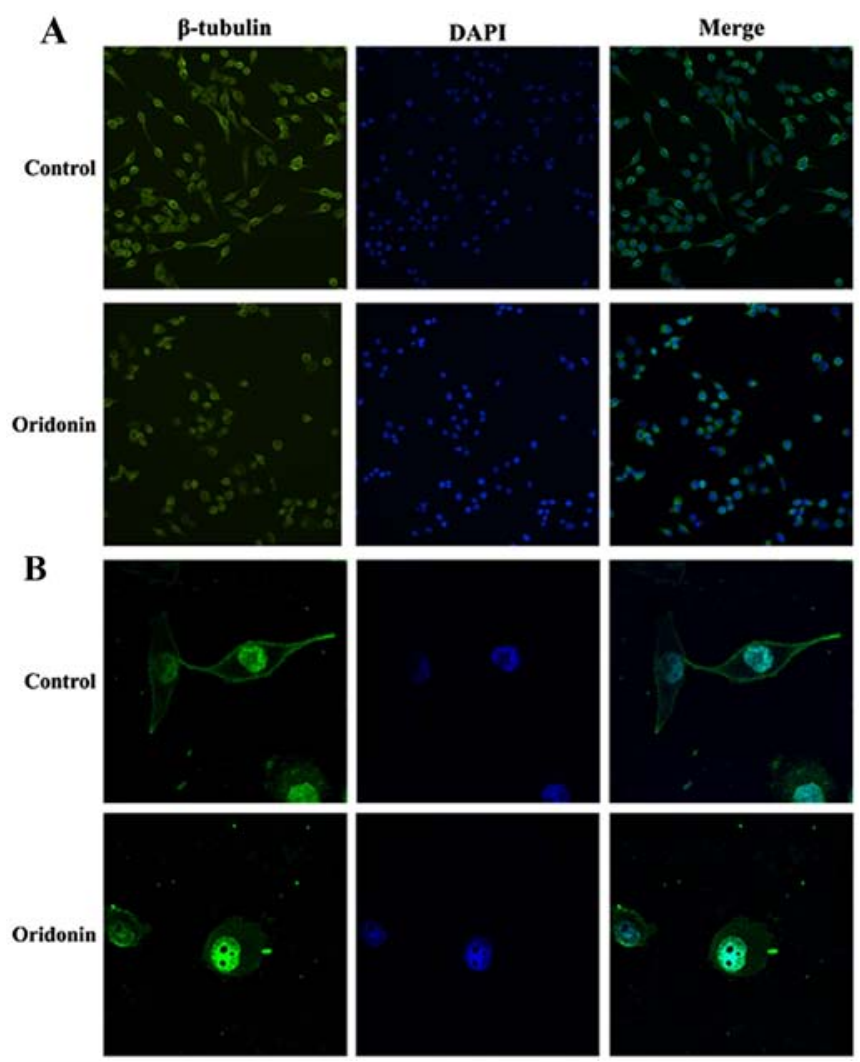

Figure 5. Oridonin changes the structure of cytoskeleton in PANC-1 cells. (A) $\beta$-tubulin magnified $\times 200$ by fluorescent microscope; (B) $\beta$-tubulin magnified x 1000 by confocal microscopy.

were found to infiltrate the membranes without Matrigel under the treatment of oridonin or miR-200b-3p, compared with untreated groups $(\mathrm{P}<0.01)$ in $\mathrm{BxPC}-3$ and PANC-1 cells.
These results indicate that oridonin or miR-200b-3p inhibits the migration of BxPC-3 and PANC-1 cells in vitro.

Oridonin reduces the expression of ICAM-1 and VCAM-1 in $B x P C-3$ and PANC-1 cells. We next investigated the effect of oridonin on intercellular adhesion molecules, including the human intercellular adhesion molecule 1 (ICAM-1) and human vascular cell adhesion molecule 1 (VCAM-1). Cells were treated with $87.8 \mu \mathrm{M}$ oridonin (BxPC-3) or $55.8 \mu \mathrm{M}$ oridonin (PANC-1) for $24 \mathrm{~h}$. The ELISA results showed that treatment of cells with oridonin reduced the expression of ICAM-1 and VCAM-1 (Fig. 4). ICAM-1 expression was significantly reduced in the oridonin treatment groups compared with the negative control group in BxPC-3 cell (94.51 \pm 2.02 vs. $152.09 \pm 2.11 \mathrm{ng} / \mathrm{l} ; \mathrm{P}<0.01)$ and PANC-1 cell $(112.66 \pm 3.21$ vs. $153.92 \pm 2.38 \mathrm{ng} / \mathrm{l} ; \mathrm{P}<0.01)$. Similar trends were observed for VCAM-1 expression, which was significantly reduced in the oridonin treatment groups compared with the negative control group in BxPC-3 cells (432.14 \pm 8.37 vs. $475.06 \pm 2.09 \mu \mathrm{g} / \mathrm{l}$; $\mathrm{P}<0.01)$ and PANC-1 cells $(471.22 \pm 4.37$ vs. $368.64 \pm 5.23 \mu \mathrm{g} / \mathrm{l}$; $\mathrm{P}<0.05)$.

Oridonin alters the structure of the cytoskeleton in PANC-1 cells. To research the effect of oridonin on cytoskeleton beta microtubule protein ( $\beta$-tubulin) in PANC-1 cells, we observed the $\beta$-tubulin in PANC- 1 cells after treatment with oridonin by immunofluorescence and confocal microscopy. As shown in the Fig. 5, PANC-1 cells are fusiform, $\beta$-tubulin are dense filiform and distributed uniformly, parallelling to the longitudinal axis of the cells. Compared with the control group, the PANC-1 cells treated with oridonin are deformed and the edges are irregular, with $\beta$-tubulin arranged disorderly, significant changes had taken place. The result illustrated that the structure of cytoskeleton in PANC-1 cells can be 
A

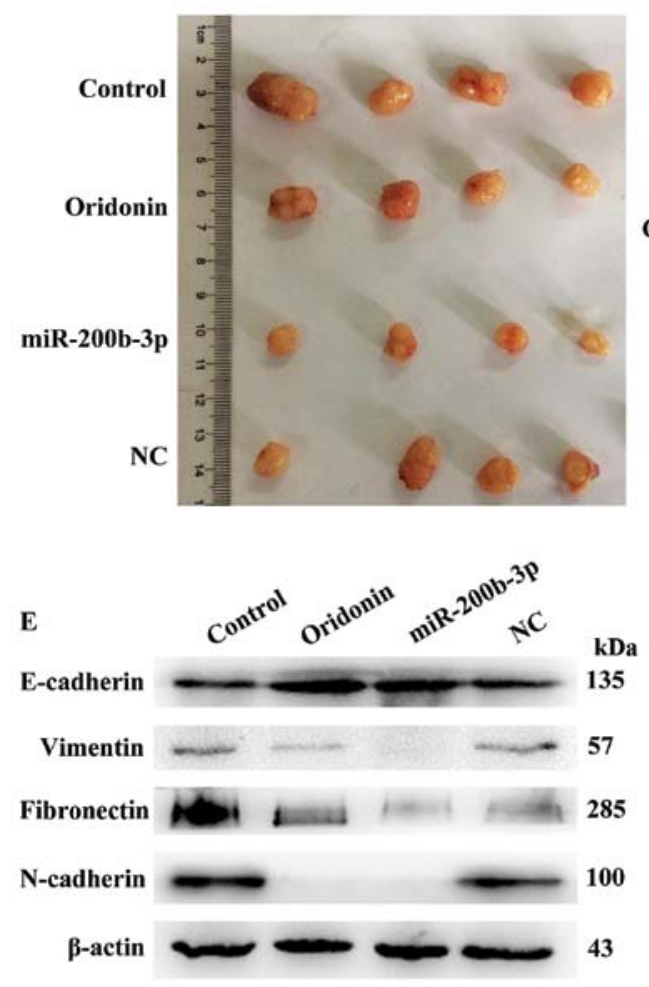

B

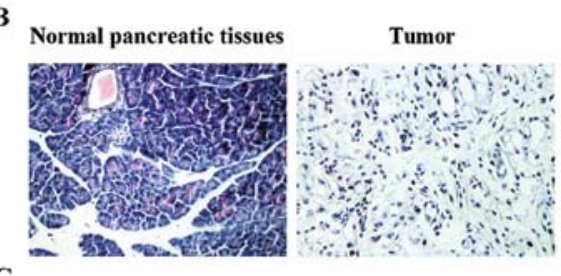

C

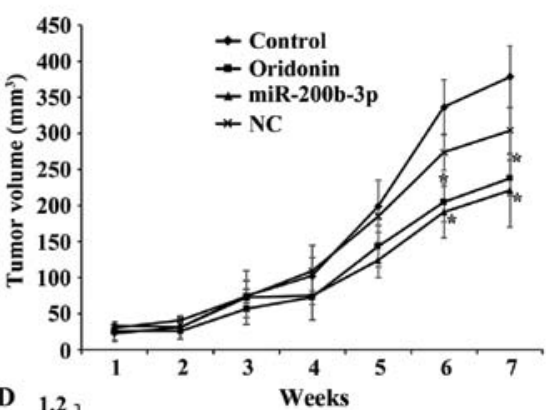

D

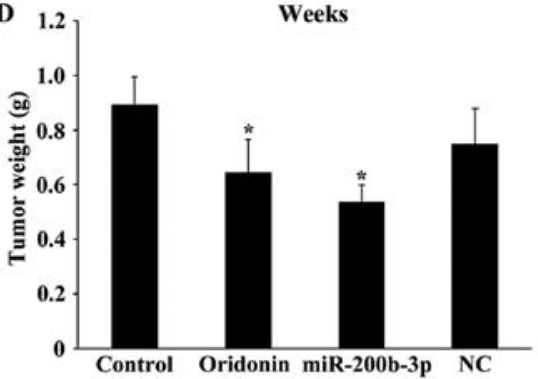

F
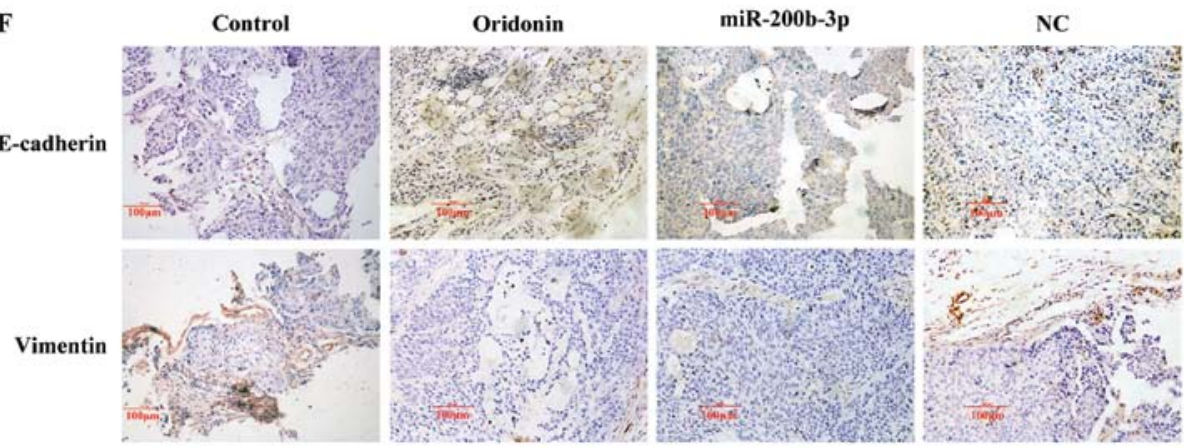

Figure 6. Oridonin/miR-200b-3p inhibits tumor growth of BxPC-3 cells and EMT in nude mice. (A) The transplantation tumors removed from nude mice; (B) the results of normal pancreatic tissues and transplantation tumor by H\&E staining; (C) tumor volumes were measured on the indicated days; (D) the weight of the transplanted tumor; (E) the protein lysates ( $40 \mu \mathrm{g} / \mathrm{lane})$ extracted from the tumor tissues were assessed by immunoblotting for E-cadherin, vimentin, fibronectin, $\mathrm{N}$-cadherin and $\beta$-actin, respectively; (F) mouse tumor tissues were immumohistochemically stained using E-cadherin and vimentin antibodies as described in Materials and methods (original magnification, $\mathrm{x} 20$; $\mathrm{P}<0.05$ vs. control.

changed by oridonin and then influence the cell morphology and function.

Oridonin/miR-200b-3p suppresses tumorigenicity and EMT in nude mice. Next, we identified the effect of oridonin/ miR-200b-3p on tumorigenicity and EMT in mouse models. The normal pancreatic tissues and transplantation tumor were stained using hematoxylin and eosin (H\&E). The result showed that the pancreatic tissues are segmented into lobules by a small amount of connective tissue separately. Acinar cells appear as grape-like cell clumps, cells presenting coneshape, nuclear is round or ovoid, located at the base of the cells, with abundant eosinophilic cytoplasm. The pancreatic duct arranges as arborization and the boundaries of the blood vessels and gland are clear. A few funicular permutations and colored shallow light islet cells distributed in the pancreatic tissue. In the heteroplastic pancreatic cancer tissue, the cell sizes show inconformity and the arrangements are irregular, acinar cells decreased significantly and cytoplasmic staining was shallow. The tissues are mainly made up of a few different ductal glands, accompanied by abundant fibrous stroma. The cell abnormality is high, columnar cells and spindle cells are mixed, no bleeding or necrosis (Fig. 6B). When injected with oridonin or BxPC-3 cells stably expressing miR-200b-3p in nude mice, the tumors grew more slowly than that of the control, and the gap expanded until the endpoint of 7-weeks (Fig. 6A). Consistently, the oridonin-treated tumors and the miR-200b-3p overexpressed tumors were smaller in size and weight compared with the control tumors (Fig. 6C and D). Together, our data indicated that oridonin/miR-200b-3p suppresses tumorigenicity in vivo. We next examined the effect of oridonin/miR-200b-3p on EMT in mouse models 
by western blot analysis. The results showed that the protein expression levels of vimentin, $\mathrm{N}$-cadherin and fibronectin were decreased while the expression of E-cadherin was increased in nude mice treated with oridonin or miR-200b-3p, comparing with the control (Fig. 6E). In addition, immunohistochemical staining for E-cadherin and vimentin in primary tumor tissues showed that the oridonin-treated group and the miR-200b-3p overexpressed group exhibited an increased expression of E-cadherin, an epithelial marker and a reduced expression of vimentin, a mesenchymal marker (Fig. 6F). These results revealed that oridonin or upregulation of $\mathrm{miR}-200 \mathrm{~b}-3 \mathrm{p}$ is associated with EMT in vivo.

\section{Discussion}

With the discovery of miRNAs, it has been shown that miRNAs are important in the regulation of gene expression networks, and they also play crucial roles in many biological processes. Abnormal expression of miRNAs is associated with many diseases, such as nervous system diseases, cardiovascular disease and cancer (20). miRNAs can function as tumor suppressors or oncogenes in cancer. To date, studies have demonstrated that there are 95 aberrant miRNAs (let-7 family, miR-7, miR-92, miR-93, miR-196a, miR-190, miR-186, miR-221, miR-222, miR-200b, miR-15b and miR-95) have been shown altered in pancreatic cancer (21).

Traditional Chinese medicines have become a widely discussed topic in relation to their potential antitumor properties. However, the mechanisms of the antitumour activity have not been completely delineated. Recently, some studies showed that the cancer may be inhibited by the active ingredients of traditional Chinese medicines though regulating miRNAs, which may be treated as targets for cancer therapies (22-24). Oridonin is one of the most effective antitumor agents obtained from Rabdosia rubescens. In the present study, the results demonstrate that oridonin can inhibit the growth of human BxPC-3 and PANC-1 pancreatic cancer cells in dosedependent manner, and the $\mathrm{IC}_{50}$ was $87.8 \mu \mathrm{M}$ in BxPC-3 cells and $55.8 \mu \mathrm{M}$ in PANC-1 cells, respectively. In our previous study, we found that oridonin alters the expression profiles of miRNAs in the BxPC-3 human pancreatic cancer cells, 105 miRNAs were significantly altered: 49 miRNAs were significantly downregulated, while 56 were significantly upregulated. Among them, miR-200b-3p was significantly downregulated (19), and also significantly downregulated in the PANC-1 cells (Fig. 1C). Next, we tried to explore the mechanism by which miR-200b-3p exerts influence on the BxPC-3 and PANC-1 cells. Zinc finger E-box-binding homeobox 1 (ZEB1) was predicted to be the theoretical target gene of miR-200b-3p using three public bioinformatic algorithms in combination (Fig. 1D).

ZEB1 is a member of the ZEB family of transcription factors, it contains two zinc finger clusters and a homeodomain (25). Growing evidence shows that ZEB1 plays an important role in cell proliferation, apoptosis, metastasis and angiogenesis (26-29). EMT is a crucial step which cancer cells must pass before they can undergo metastasis and ZEB1 is one of the key inducers of epithelial-to-mesenchymal transition. In the present study, we found that overexpression of miR-200b-3p significantly downregulated the expression of
ZEB1 protein, which demonstrated that ZEB1 is one of the target genes of miR-200b-3p. Whereas the expression of miR-200b-3p and ZEB1 were all downregulated by oridonin in the BxPC-3 and PANC-1 cells. These results demonstrate that the relationship among oridonin, miR-200b-3p and ZEB1 is not a single cascade relationship, which may be affected by other genes and signal pathways. It remains to be investigated in our further study.

The miR-200 family is composed of miR-141, -200a, $-200 b,-200 c,-429$ and miR-205. Kundu et al (30) found that the miR-200 family and the miR-183-96-182 cluster can target Foxf2 to inhibit EMT, invasion and metastasis in lung cancers. In anaplastic thyroid cancer, miR-200 involves in epithelial-to-mesenchymal transition (EMT) by regulating EGF/EGFR signaling (31). A recent study has shown that Pien Tze Huang can inhibit metastasis of human colorectal carcinoma cells via modulating TGF- $\beta 1 / \mathrm{ZEB} / \mathrm{miR}-200$ signaling network (32). The miR-200 family has been demonstrated as EMT-suppressive miRNAs in many types of cancer such as triple-negative breast cancer (33), bladder cancer cells (34) and prostate cancer (35). To date, the study of pancreatic cancer has demonstrated that miR-200a (36) and miR-141 (37) can inhibit invasion and migration, miR$200 \mathrm{c}$ is a prognostic biomarker (38), miR-429 determines poor outcome and inhibits cells growth (39), but there are few reports on miR-200b and EMT. In the present study, we demonstrate that overexpression of miR-200b-3p significantly inhibits the expression of ZEB1, N-cadherin, fibronectin and increases the expression of E-cadherin (Fig. 2). These data demonstrate that miR-200b-3p could inhibit EMT. To further study the effect of oridonin/ miR-200b-3p in inhibiting cell migration in the BxPC-3 and PANC-1 cells, Transwell migration assay was carried out in 24-well modified chambers precoated without Matrigel. The result showed that oridonin or miR-200b-3p can inhibit the migration of BxPC-3 and PANC-1 cells in vitro (Fig. 3). But as shown in the Fig. 2, oridonin significantly inhibits the expression of ZEB1, N-cadherin, fibronectin, but did not increase the expression of E-cadherin. This result illustrates that oridonin could the expression of the mesenchymal markers, but could not increase the expression of the epithelial markers. The reason may be that the expression of E-cadherin is regulated by many other genes and the inhibition of migration by oridonin is not mainly through type III EMT in the BxPC-3 and PANC-1 cells.

The type II EMT is characterized by the differentiation of epithelial cells into new fibroblast-like cells and associated with inflammation in wound repair, tissue regeneration or organ fibrosis. In the process of organ fibrosis, type II EMT can continue to respond to ongoing inflammation, leading eventually to organ fibrosis, which is in essence an unabated form of wound healing due to persistent inflammation (15). Rabdosia rubescens is characterized as heat-clearing, detoxifying, antiinflammatory and anti-nociceptive. The pancreatic cancer is related to inflammation. In our previous study, we found that inflammatory factors (IL-1 $\beta$, IL-6 and IL-33) were decreased in BxPC-3 cells treated with oridonin, and oridonin also regulated nuclear transcription factor pathways (40). In addition, TGF- $\beta 1$ induced EMT could be enhanced by inflammatory factors (TNF- $\alpha$ and IL-1 $\beta$ ) in human bronchial epithelial 
cells $(41,42)$. Therefore, oridonin may inhibit the type II EMT through its anti-inflammatory effects.

Fibronectin (FN), a glycoprotein with various biological activity, is one of the important extracellular matrix proteins in the type II EMT, which are involved in cellular adhesion and migration processes (43). FN could drive angiogenesis, metastasis and chemoresistance in pancreatic ductal adenocarcinoma (44). The metastasis of tumors is dependent on angiogenesis and connected with the vascular endothelial growth factor (VEGF). In our previous study, we found that oridonin specifically inhibits the expression of VEGF in a dose-dependent manner. In addition, this study has shown that oridonin also specifically inhibits the expression of fibronectin (Fig. 2). Thus, it presumed that the migration of pancreatic cancer may be inhibited by oridonin though inhibiting angiogenesis and fibrosis to inhibit the type II EMT.

Cell adhesion molecule is a variety of biological activities of transmembrane glycoprotein, produced by cells to mediate the interaction of cell-cell and cell-matrix, including immunoglobulin superfamily. Intercellular adhesion molecule-1 (ICAM-1) and vascular cell adhesion molecule-1 (VCAM-1) are the members of the immunoglobulin superfamily. ICAM-1 is a cell surface glycoprotein and plays an important role in the tumor cell expansion or metastasis. A previous report showed that silencing of ICAM-1 can inhibit the metastatic ability in human breast cancer cell lines significantly (45). Amphiregulin can enhance the expression of ICAM-1 and promote tumor metastasis in human osteosarcoma (46). Osteoblast-derived WISP-1 promotes migration and VCAM-1 expression in human prostate cancer cells by downregulating miR-126 expression via $\alpha v \beta 1$ integrin, FAK and p38 signaling pathways (47). Our results indicate that ICAM-1 and VCAM-1 were decreased in the BxPC-3 and PANC-1 cells by oridonin treatment which may be the mechanism of inhibition of migration (Fig. 4).

Cytoskeleton is mainly represented by microfilaments, intermediate filaments and microtubules and are also associated with cellular shape and various cellular functions (48). The actin proteins are the structural component of microfilament to constitute the cytoskeleton of cells. Previous studies have shown that F-actin filaments contributed to cell migration in MDA-MB-231 cells (49). Prolactin could promote the migration of breast cancer cells through remodeling the actin cytoskeleton (50). The microtubules are composed of $\alpha$-tubulin and $\beta$-tubulin, which have important functions including maintenance of cell morphology, cellular signaling, cell migration and formation of cell polarity (51). The results suggest that cytoskeleton is closely related to tumor migration. To assess whether oridonin have impact on the cytoskeleton in PANC-1 cell, we observed $\beta$-tubulin in PANC-1 cells after treatment with oridonin by immunofluorescence and confocal microscopy. As shown in Fig. 5 , oridonin can significantly change the cytoskeleton of PANC-1 cells, which may be related to its inhibition of migration in pancreatic cancer.

To extend the in vitro observations, in vivo experiments were performed by tumor formation assay in nude mice. Our results demonstrated that oridonin/miR-200b-3p suppressed tumorigenicity and EMT in vivo. In this study, we found that oridonin significantly inhibited the expression of E-cadherin in the BxPC-3 cells and PANC-1 cells (Fig. 2), but increased the expression of E-cadherin in nude mice (Fig. 6E). The mechanisms are not clear. Recently, some studies showed that the expression of E-cadherin could be regulated by stromal cells, which is an important part of tumor microenvironment. Thus, this may be the reason that the expression of E-cadherin was inhibited in the BxPC-3 cells and PANC-1 cells, but increased in nude mice. Taken together, it indicated that EMT might be an ideal target for cancer therapy not only in epithelial cancer cells, but also in pancreatic cancer.

In conclusion, our data suggest that oridonin was able to inhibit the proliferation and migration of BxPC-3 and PANC-1 cells. miR-200b-3p is downregulated in BxPC-3 and PANC-1 cells by oridonin, and functions as a novel tumor suppressor to regulate pancreatic cancer cell migration through inhibiting the EMT. The novel mechanisms of oridonin mediated by decreasing cancer migration is not through III-EMT by miR-200-3p/ZEB1 axis, and may be related to other miRNAs, II-EMT, or altering the cytoskeleton. In addition, oridonin/ miR-200b-3p inhibited pancreatic cancer growth and EMT in nude mice. Thus, oridonin and miR-200b-3p may be useful in the therapy of pancreatic cancer.

\section{Acknowledgements}

The present study was supported by the Zhejiang Province Project of Science Technology Department (no. 2014C33265), the Natural Science Foundation of Zhejiang Province (no. LY14H160037) and the Zhejiang Medical Technology and Education (no. 2015KYB255).

\section{References}

1. Li D, Xie K, Wolff R and Abbruzzese JL: Pancreatic cancer. Lancet 363: 1049-1057, 2004

2. Yokoyama Y, Nimura Y and Nagino M: Advances in the treatment of pancreatic cancer: Limitations of surgery and evaluation of new therapeutic strategies. Surg Today 39: 466-475, 2009.

3. Sun HD, Huang SX and Han QB: Diterpenoids from Isodon species and their biological activities. Nat Prod Rep 23: 673-698, 2006.

4. Wu QX, Yuan SX, Ren CM, Yu Y, Sun WJ, He BC and Wu K: Oridonin upregulates PTEN through activating p38 MAPK and inhibits proliferation in human colon cancer cells. Oncol Rep 35: 3341-3348, 2016.

5. Gao S, Tan H, Zhu N, Gao H, Lv C, Gang J and Ji Y: Oridonin induces apoptosis through the mitochondrial pathway in human gastric cancer SGC-7901 cells. Int J Oncol 48: $2453-$ 2460, 2016.

6. Zhang XH, Liu YX, Jia M, Han JS, Zhao M, Ji SP and Li AM: Oridonin inhibits tumor growth in glioma by inducing cell cycle arrest and apoptosis. Cell Mol Biol 60: 29-36, 2014.

7. Dong Y, Zhang T, Li J, Deng H, Song Y, Zhai D, Peng Y, Lu X, Liu M, Zhao Y, et al: Oridonin inhibits tumor growth and metastasis through anti-angiogenesis by blocking the Notch signaling. PLoS One 9: e113830, 2014.

8. Bartel DP: MicroRNAs: Target recognition and regulatory functions. Cell 136: 215-233, 2009.

9. Ambros V: The functions of animal microRNAs. Nature 431: 350-355, 2004.

10. Kozomara A and Griffiths-Jones S: miRBase: Integrating microRNA annotation and deep-sequencing data. Nucleic Acids Res 39 (Database): D152-D157, 2011.

11. Zidar N, Boštjančič E, Jerala M, Kojc N, Drobne D, Štabuc B and Glavač D: Down-regulation of microRNAs of the miR-200 family and up-regulation of Snail and Slug in inflammatory bowel diseases - hallmark of epithelial-mesenchymal transition. J Cell Mol Med 20: 1813-1820, 2016. 
12. Chung VY, Tan TZ, Tan M, Wong MK, Kuay KT, Yang Z, Ye J, Muller J, Koh CM, Guccione E, et al: GRHL2-miR-200-ZEB1 maintains the epithelial status of ovarian cancer through transcriptional regulation and histone modification. Sci Rep 6: 19943, 2016.

13. Thiery JP: Epithelial-mesenchymal transitions in tumour progression. Nat Rev Cancer 2: 442-454, 2002.

14. Cervantes-Arias A, Pang LY and Argyle DJ: Epithelialmesenchymal transition as a fundamental mechanism underlying the cancer phenotype. Vet Comp Oncol 11: 169-184, 2013.

15. Kalluri R and Weinberg RA: The basics of epithelial-mesenchymal transition. J Clin Invest 119: 1420-1428, 2009.

16. Zeng F, Xue M, Xiao T, Li Y, Xiao S, Jiang B and Ren C: MiR-200b promotes the cell proliferation and metastasis of cervical cancer by inhibiting FOXG1. Biomed Pharmacother 79: 294-301, 2016

17. Wu H, Wang G, Wang Z, An S, Ye P and Luo S: A negative feedback loop between miR-200b and the NF-kappaB pathway via IKBKB/IKK-beta in breast cancer cells. FEBS J 283: 2259-2271 2015 .

18. Zhang HF, Alshareef A, Wu C, Li S, Jiao JW, Cao HH, Lai R, $\mathrm{Xu}$ LY and Li EM: Loss of miR-200b promotes invasion via activating the Kindlin-2/integrin $\beta 1 / \mathrm{AKT}$ pathway in esophageal squamous cell carcinoma: An E-cadherin-independent mechanism. Oncotarget 6: 28949-28960, 2015.

19. Gui Z, Li S, Liu X, Xu B and Xu J: Oridonin alters the expression profiles of microRNAs in BxPC-3 human pancreatic cancer cells. BMC Complement Altern Med 15: 117, 2015.

20. Lu J, Getz G, Miska EA, Alvarez-Saavedra E, Lamb J, Peck D, Sweet-Cordero A, Ebert BL, Mak RH, Ferrando AA, et al: MicroRNA expression profiles classify human cancers. Nature 435: 834-838, 2005.

21. Zhang Y, Li M, Wang H, Fisher WE, Lin PH, Yao Q and Chen C: Profiling of 95 microRNAs in pancreatic cancer cell lines and surgical specimens by real-time PCR analysis. World J Surg 33: 698-709, 2009

22. Sun M, Estrov Z, Ji Y, Coombes KR, Harris DH and Kurzrock R Curcumin (diferuloylmethane) alters the expression profiles of microRNAs in human pancreatic cancer cells. Mol Cancer Ther 7: 464-473, 2008.

23. Sakurai MA, Ozaki Y, Okuzaki D, Naito Y, Sasakura T, Okamoto A, Tabara H, Inoue T, Hagiyama M, Ito A, et al: Gefitinib and luteolin cause growth arrest of human prostate cancer PC-3 cells via inhibition of cyclin G-associated kinase and induction of miR-630. PLoS One 9: e100124, 2014.

24. Wu N, Wu GC, Hu R, Li M and Feng H: Ginsenoside Rh2 inhibits glioma cell proliferation by targeting microRNA-128. Acta Pharmacol Sin 32: 345-353, 2011.

25. Zhang Y, Liu G, Wu S, Jiang F, Xie J and Wang Y: Zinc finger E-box-binding homeobox 1: Its clinical significance and functional role in human thyroid cancer. Onco Targets Ther 9 : 1303-1310, 2016.

26. Li YJ, Ping C, Tang J and Zhang W: MicroRNA-455 suppresses non-small cell lung cancer through targeting ZEB1. Cell Biol Int 40: 621-628, 2016

27. Hou L, Li Q, Yu Y, Li M and Zhang D: SET8 induces epithelial-mesenchymal transition and enhances prostate cancer cell metastasis by cooperating with ZEB1. Mol Med Rep 13: $1681-1688,2016$

28. Zhang G, An H and Fang X: MicroRNA-144 regulates proliferation, invasion, and apoptosis of cells in malignant solitary pulmonary nodule via zinc finger E-box-binding homeobox 1. Int J Clin Exp Pathol 8: 5960-5967, 2015.

29. Inuzuka T, Tsuda M, Kawaguchi $H$ and Ohba $Y$ : Transcription factor 8 activates R-Ras to regulate angiogenesis. Biochem Biophys Res Commun 379: 510-513, 2009.

30. Kundu ST, Byers LA, Peng DH, Roybal JD, Diao L, Wang J, Tong P, Creighton CJ and Gibbons DL: The miR-200 family and the miR-183 96 182 cluster target Foxf 2 to inhibit invasion and metastasis in lung cancers. Oncogene 35: 173-186, 2016.

31. Xue L, Su D, Li D, Gao W, Yuan R and Pang W: MiR-200 regulates epithelial-mesenchymal transition in anaplastic thyroid cancer via EGF/EGFR signaling. Cell Biochem Biophys 72: 185-190, 2015

32. Shen A, Lin W, Chen Y, Liu L, Chen H, Zhuang Q, Lin J, Sferra TJ and Peng J: Pien Tze Huang inhibits metastasis of human colorectal carcinoma cells via modulation of TGF- $\beta 1 /$ ZEB/miR-200 signaling network. Int J Oncol 46: 685-690, 2015.
33. Rhodes LV, Martin EC, Segar HC, Miller DF, Buechlein A, Rusch DB, Nephew KP, Burow ME and Collins-Burow BM: Dual regulation by microRNA-200b-3p and microRNA-200b-5p in the inhibition of epithelial-to-mesenchymal transition in triple-negative breast cancer. Oncotarget 6: 16638-16652, 2015.

34. Liu L, Qiu M, Tan G, Liang Z, Qin Y, Chen L, Chen H and Liu J: miR-200c inhibits invasion, migration and proliferation of bladder cancer cells through down-regulation of BMI-1 and E2F3. J Transl Med 12: 305, 2014.

35. Williams LV, Veliceasa D, Vinokour E and Volpert OV: miR-200b inhibits prostate cancer EMT, growth and metastasis. PLoS One 8: e83991, 2013.

36. Lu Y, Lu J, Li X, Zhu H, Fan X, Zhu S, Wang Y, Guo Q, Wang L, Huang Y, et al: MiR-200a inhibits epithelial-mesenchymal transition of pancreatic cancer stem cell. BMC Cancer 14: 85, 2014.

37. Xu L, Li Q, Xu D, Wang Q, An Y, Du Q, Zhang J, Zhu Y and Miao Y: hsa-miR-141 downregulates TM4SF1 to inhibit pancreatic cancer cell invasion and migration. Int J Oncol 44: 459-466, 2014.

38. Paik WH, Song BJ, Kim HW, Kim HR and Hwang JH: MicroRNA-200c as a prognostic biomarker for pancreatic cancer. Korean J Gastroenterol 66: 215-220, 2015.

39. Song B, Zheng K, Ma H, Liu A, Jing W, Shao C, Li G and Jin G: miR-429 determines poor outcome and inhibits pancreatic ductal adenocarcinoma growth by targeting TBK1. Cell Physiol Biochem 35: 1846-1856, 2015.

40. Chen RY, Xu B, Chen SF, Chen SS, Zhang T, Ren J and Xu J: Effect of oridonin-mediated hallmark changes on inflammatory pathways in human pancreatic cancer (BxPC-3) cells. World J Gastroenterol 20: 14895-14903, 2014.

41. Kamitani S, Yamauchi Y, Kawasaki S, Takami K, Takizawa H, Nagase T and Kohyama T: Simultaneous stimulation with TGF- $\beta 1$ and TNF- $\alpha$ induces epithelial mesenchymal transition in bronchial epithelial cells. Int Arch Allergy Immunol 155: $119-128,2011$.

42. Doerner AM and Zuraw BL: TGF-betal induced epithelial to mesenchymal transition (EMT) in human bronchial epithelial cells is enhanced by IL-1beta but not abrogated by corticosteroids. Respir Res 10: 100, 2009.

43. Wilson CB, Leopard J, Cheresh DA and Nakamura RM: Extracellular matrix and integrin composition of the normal bladder wall. World J Urol 14 (Suppl 1): S30-S37, 1996.

44. Topalovski M and Brekken RA: Matrix control of pancreatic cancer: New insights into fibronectin signaling. Cancer Lett 381: 252-258, 2015.

45. Di D, Chen L, Wang L, Sun P,Liu Y, Xu Zand Ju J: Downregulation of human intercellular adhesion molecule-1 attenuates the metastatic ability in human breast cancer cell lines. Oncol Rep 35: 1541-1548, 2016.

46. Liu JF, Tsao YT and Hou CH: Amphiregulin enhances intercellular adhesion molecule-1 expression and promotes tumor metastasis in human osteosarcoma. Oncotarget 6: 40880-40895, 2015.

47. Tai HC, Chang AC, Yu HJ, Huang CY, Tsai YC, Lai YW, Sun HL, Tang $\mathrm{CH}$ and Wang SW: Osteoblast-derived WNT-induced secreted protein 1 increases VCAM-1 expression and enhances prostate cancer metastasis by down-regulating miR-126. Oncotarget 5: 7589-7598, 2014.

48. Theurkauf WE, Smiley S, Wong ML and Alberts BM Reorganization of the cytoskeleton during Drosophila oogenesis: Implications for axis specification and intercellular transport. Development 115: 923-936, 1992.

49. Wu L, Wang X, Liu Q, Wingnang Leung A, Wang P and Xu C: Sinoporphyrin sodium mediated photodynamic therapy inhibits the migration associated with collapse of F-actin filaments cytoskeleton in MDA-MB-231 cells. Photodiagnosis Photodyn Ther 13: 58-65, 2015.

50. da Silva PL, do Amaral VC, Gabrielli V, Montt Guevara MM, Mannella P, Baracat EC, Soares-Jr JM and Simoncini T: Prolactin promotes breast bancer bell migration through actin cytoskeleton remodeling. Front Endocrinol (Lausanne) 6: 186, 2015.

51. Li L and Yang XJ: Tubulin acetylation: Responsible enzymes, biological functions and human diseases. Cell Mol Life Sci 72: 4237-4255, 2015. 